Article

\title{
Geometric Objects: A Quality Index to Electromagnetic Energy Transfer Performance in Sustainable Smart Buildings
}

\author{
Juan C. Bravo@ and Manuel V. Castilla * \\ Higher Polytechnic School, University of Seville, Seville 41011, Spain; carlos_bravo@us.es \\ * Correspondence: mviggo@us.es; Tel.: +34-954-556-440
}

Received: 29 October 2018; Accepted: 28 November 2018; Published: 29 November 2018

\begin{abstract}
Sustainable smart buildings play an essential role in terms of more efficient energy. However, these buildings as electric loads are affected by an important distortion in the current and voltage waveforms caused by the increasing proliferation of nonlinear electronic devices. Overall, buildings all around the world consume a significant amount of energy, which is about one-third of the total primary energy resources. Optimization of the power transfer process of such amount of energy is a crucial issue that needs specific tools to integrate energy-efficient behaviour throughout the grid. When nonlinear loads are present, new capable ways of thinking are needed to consider the effects of harmonics and related power components. In this manner, technology innovations are necessary to update the power factor concept to a generalized total or a true one, where different power components involved in it calculation, properly reflect each harmonic interaction. This work addresses an innovative theory that applies the Poynting Vector philosophy via Geometric Algebra to the electromagnetic energy transfer process providing a physical foundation. In this framework, it is possible to analyse and detect the nature of disturbing loads in the exponential growth of new globalized buildings and architectures in our era. This new insight is based on the concept of geometric objects with different dimension: vector, bivector, trivector, multivector. Within this paper, these objects are correlated with the electromagnetic quantities responsible for the energy flow supplied to the most common loads in sustainable smart buildings. Besides, it must be considered that these phenomena are characterized by a quality index multivector appropriate even for detecting harmonic sources. A numerical example is used to illustrate the clear capabilities of the suggested index when it applies to industrial loads for optimization of energy control systems and enhance comfort management in smart sustainable buildings.
\end{abstract}

Keywords: smart building; harmonics; geometric algebra; Poynting Multivector

\section{Introduction}

\subsection{Motivation}

Nowadays, professional and academic experts have started to consider the term "Smart Sustainable Cities" [1] so as to incorporate the different aspects of sustainability in the classical "smart cities" new concept. In fact, literature tends to consider a sustainable city as a whole place of sustainable smart buildings that have a strong environmental focus with a balance within the buildings and the city between infrastructures, information and communications technology (ICT), smart technologies, and urban metabolism, focusing mainly on consumption and energy saving [2].

Buildings all around the world consume a significant amount of energy, which is about one-third of the total primary energy resources [3]. For this reason, building energy efficiency has turned out 
to be a multi-faceted problem and the majority of harmonic problems affecting sustainable smart buildings are generated within new applications and grids of global architectures. This is due, in part, to a proliferation of non-linear loads connected to the networks of the building. These technologies as CCTV recognition systems, automatic smart air conditioning equipment, artificial intelligence (AI), the latest generation of computers, all types of smart detectors and warning systems, and other power electronic equipment are the main sources of such problems. The result of using such highly non-linear load is that the current waveform is distorted. Thus, causing an excessive harmonic of current and voltage. Besides the proximity of many of this new smart building category (industrial and residential smart constructions) will contribute to the distortion of the electric power quality of the feeder, which supplies these constructions and new architectures. These harmonics can cause serious problems in power systems, excessive heat of appliances and machines, aging of electronic component and a decreased capacity, failures of the safety devices and measures of protection, lower power factor and consequently, a reducing power system efficiency due to increasing losses. All these effects are some of the main results of harmonics in power distribution systems. Note, that Harmonic distortion can cause significant costs in distribution networks. Harmonic cost consists of harmonic energy losses, premature aging and de-rating of electrical equipment. The difference between the known generation and the estimated consumption is considered as the energy loss.

Other causes of energy loss and, consequently, an increase in the cost of it, are due to the lack of control of energy efficiency in the thermal performance of buildings and energy balance. Most of the building heat losses occur through the building envelope. In recent years, an important number of papers on quantification and optimization of energy efficiency in buildings has been published referring to the standard for buildings. Many of these works have been developed in different areas of application such energy losses in the building envelope as HVAC systems (heating, ventilation and air conditioning), windows, etc. All these works have in common the aim of making efficient buildings from an energy point of view to be sustainable. Particularly, in [4], an original approach for the U-value evaluation (analogies with coeval buildings, the calculation method, the in-situ measurements and the laboratory tests) is taken. In [5], measures of energy efficiency and optimization in the building sector are also evaluated.

In this article, it is proposed an energy quality multivector index (EQI) based on the Generalized Poynting Multivector (GPM) theory, that possesses clear advantages from the viewpoint of harmonic sources detection and minimization in sustainable smart buildings.

\subsection{Literature Review}

Valuable contributions in electromagnetic field applied to the electric power theory analysis under multi-sinusoidal conditions have been appeared so far in the literature. Despite they have different interpretations, most of them share the common denominator of dealing with the suitability of the Poynting Vector to explain the electromagnetic energy flow in electric systems [6].

In sinusoidal systems, Complex Algebra provides an appropriate framework to analyse the relationship between the Complex Poynting Vector and the energy flow [7].

In one-ports under periodic multi-sinusoidal linear/non-linear operation this issue has still some fundamental unsolved aspects. Nevertheless, some progress has unquestionably been made from numerous valuable contributions in the literature [8-21], each one of them trying to clarify different aspects of the problem by applying the classic Poynting vector (PV) concept. Among them $[8,9,14,15]$ masterfully explain the power factor concept and the physical mechanism of energy propagation in electric power systems; Ferrero et al. in [17] reconsiders the physical meaning assigned to the non-active components of the Park instantaneous power; Balci et al. in [20] describes the transition between PV and instantaneous active and reactive powers; and Faria et al. in [11] computes the instantaneous power directly from Maxwell's equations together with the evaluation of the PV flux. On the other hand, several applications are given by means of PV: Lundin et al. in [10] analyses synchronous generators using field simulations; De León et al. in [12] identifies terms in nonlinear-switched circuits; 
Cheng et al. in [13] calculates the reactive power of iced transmission line; Todeschini et al. in [16] detects and explains the process of compensation and restoration of symmetry in an unbalanced system and Stahlhut et al. in [21] examines critically the PV possibilities in the area of instrumentation of losses. However, critics of PV calculations $[18,19]$ argue that electromagnetic theory is useless for practical applications of electric power theory. Nevertheless, the large number of papers published on the physical and/or mathematical nature of electromagnetic energy transfer suggest that the work has not been finished.

The multidimensionality of power equation and energy quality indexes in the multi-sinusoidal case is the underlying obstacle that considerably complicates the issue at hand. Instead, reference [13] is a pioneering contribution to the role of a Poynting Multivector to the interpretation of the energy flows based on an original Clifford Vector space. Accordingly, this work adds a new representation of electromagnetic power theory deduced from a Generalized Poynting Multivector [22].

\subsection{Contribution}

An introduction to "geometric objects" in Geometric Algebra (GA) and the associated phenomena within the electrical systems is developed. In addition, these entities permit a unified treatment of the energy flow concept. By means of the GA framework, the classic explanations of the energy flow process based on interactions between electric and magnetic fields of like-frequency is overcome. The proposed generalization adds the cross-fields interactions in a natural manner, as well.

By this way, this paper is concerned to the application of the Generalized Poynting Multivector (GPM) concept [22] to provide both of them, a physical foundation to the non-active electromagnetic geometric objects as well as a new multivector index to assess the efficiency of the complete energy process in sustainable and smart building loads supplied from a transmission line.

\section{Geometric Objects}

Geometric Algebra [23-26] is based on the concept of objects with different geometrical dimension that result from the geometric product of distinct graded basis elements, e.g., scalars, vectors, 2-vectors and so on. Thus, starting from vectors within an n-dimensional linear space over the real numbers $\mathcal{V}^{n}$, the geometric product of vectors $a b$ if $a, b \in \mathcal{V}^{n}$ is formed by a symmetric inner product

$$
a \cdot b=\frac{1}{2}(a b+b a)
$$

and an antisymmetric outer product

$$
a \wedge b=\frac{1}{2}(a b-b a)
$$

Therefore, $a b$ has the canonical decomposition

$$
\widetilde{M}=a b=a \cdot b+a \wedge b
$$

The resulting multivector $\widetilde{M}$ is the sum of a scalar $(a \cdot b)$ and a bivector or 2-vector $(a \wedge b)$ object. Despite this sum of two distinct objects might seem strange at first sight and it is against the common rule that only same objects should be added, this Clifford's brilliant idea [23] allows to generalize easily the product to arbitrary higher dimensions and incorporates geometric interpretations of objects and operators.

Thus, a bivector can be viewed as directed plane segment, in much the same way as a vector represents a directed line segment, Figure 1. The bivector $a \wedge b$ has a magnitude $|a \wedge b|$ equal to the usual scalar area of the circle in Figure 1, with the direction of the plane in which the circle lies, and with sense, which can be assigned to the circle in the plane. Then, just as a vector $a$ represents (or is represented by) a directed line segment and a bivector $a \wedge b$ represents a directed plane segment, the trivector (3-vector) $a \wedge b \wedge c$ is a grade-3 object that represents a volume (the sphere). 
In $\mathcal{G}_{3}$, the geometric algebra of three-dimensional space, a general multivector can be written as

$$
\widetilde{M}=\alpha+\lambda+B+\mu J
$$

where $a$ is a vector, $B$ is a bivector, $I$ is a trivector (pseudoscalar) and $\lambda$ and $\mu$ are both scalars. The three orthogonal basis vectors $\left\{\sigma_{1}, \sigma_{2}, \sigma_{3}\right\}$, the three bivectors $\left\{\sigma_{1} \sigma_{2}, \sigma_{2} \sigma_{3}, \sigma_{3} \sigma_{1}\right\}$, the unit scalar, and the trivector $\sigma_{1} \sigma_{2} \sigma_{3}$ define a graded linear space of total dimension $8=2^{3}$ and are shown in Figure 1.

The unit right-handed pseudoscalar for the space $J$ squares to $-1, J^{2}=-1$. The pseudoscalar, as well as bivectors, change sign under reversion, but vectors do not. This reversion operation reverses the order of vectors in any product and the convention. So, the reverse of multivector $\widetilde{M}$ derived only from vectors

$$
\widetilde{M}^{\dagger}=b a=a \cdot b-a \wedge b
$$

or in the general form resulting from all the possible basis product in $\mathcal{G}_{3}$

$$
\widetilde{M}^{+}=\alpha+\lambda-B-\mu J
$$

For all the formulae presented below the followed notation uses the tilde $\sim$ for multivectors, Euclidean vectors are written in bold font, the dagger symbol + denotes the reverse operation and the upper asterisk * represents the complex conjugate operation. For a complete understanding of notation, a list of symbols is summarized in the glossary.
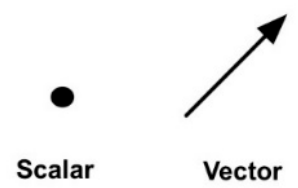

Vector

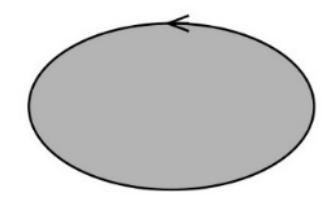

Bivector

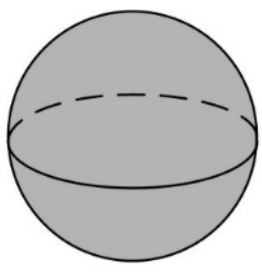

Trivector

Figure 1. Geometric objects.

\section{The System Model}

An "electrical system" is shown in Figure 2 and it can be considered as a space confined by a closed surface to/from which electric power is supplied/received by conductors carrying an electric current. The total instantaneous power transmitted is

$$
s(t)=\sum_{k} u_{k} i_{k}
$$

Two main electric power processes occur in the system:

- $\quad$ Electric power (energy) is dissipated.

- Electric power (energy) in the system derives from an electric field and magnetic field interactions.

In a general form, the electromagnetic phenomena occurring in the system under study can be roughly modelled by electric circuits.

Despite the application of GA to circuit analysis and power theory has a very short history some relevant advances have been made in this area from different perspectives [27-35]. 


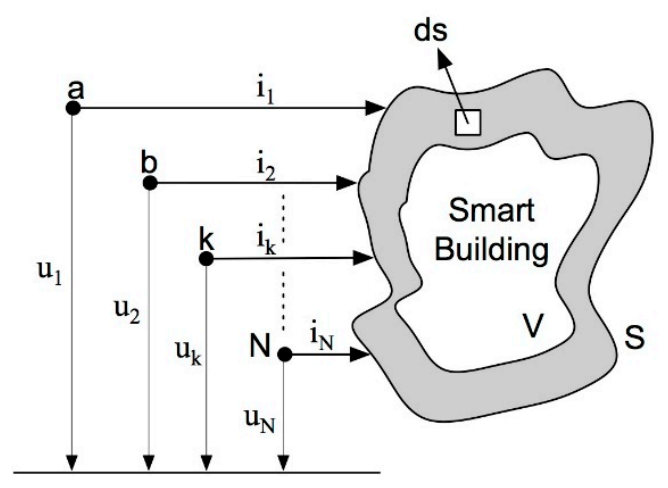

Figure 2. Electrical system.

\section{Generalized Poynting Multivector}

In this work, space coordinates and time (frequency) coordinates have a differentiated treatment. Space domain remains represented by the classical Euclidean approach, the harmonic field in both, time and frequency domains, are characterized by complex geometric algebra, $\mathcal{C} \mathcal{G}_{n}$. The complete space-time framework results in the hybrid $\mathcal{C G}_{n}^{t}-\mathbb{R}^{3}$ structure named Generalized Euclidean Space $\left(\mathcal{C G}_{n}-\mathbb{R}^{3}\right.$ for space-frequency domain) [22].

In this $\mathcal{C G}_{n}-\mathbb{R}^{3}$ algebraic approach, the Generalized Poynting Multivector (GPM) is defined as

$$
\widetilde{\boldsymbol{\Pi}}=\sum_{p, q}\left(\widetilde{\boldsymbol{E}}_{p} \odot \widetilde{\boldsymbol{H}}_{q}^{*}\right)
$$

where $\widetilde{\boldsymbol{E}}_{\boldsymbol{p}}$ and $\widetilde{\boldsymbol{H}}_{\boldsymbol{q}}$ vectors are called 'spatial geometric phasors' of the electric and magnetic harmonic fields, $\widetilde{\boldsymbol{H}}_{\boldsymbol{q}}^{*}$ vector is the conjugate of the q-th harmonic spatial geometric phasor $\widetilde{\boldsymbol{H}}_{\boldsymbol{q}}$ and ' $\odot$ ' is the generalized geometric product (GGP) [27].

From this multivectorial field theory, the flux of the GPM quantity for a volume $v$ enclosed by a surface $\varsigma$, (GPM theorem), can be expanded into two terms.

$$
\iint_{\zeta} n \cdot \widetilde{\Pi} d \zeta=\iint_{\zeta} \sum_{p} n \cdot \widetilde{\mathcal{P}}_{p} d \zeta+\iint_{\zeta} \sum_{p \neq q} n \cdot \widetilde{\mathcal{D}}_{p q} d \zeta=\widetilde{S}
$$

where $n$ is the unitary vector orthogonal to the infinitesimal surface $d \varsigma, \widetilde{\mathcal{P}}$ is the Poynting Multivector (PM) and $\widetilde{\mathcal{D}}$ is the Complementary Poynting Multivector $(\mathrm{CPM})$ and $\widetilde{S}=\widetilde{U} \odot \widetilde{I}^{*}$ is the Power Multivector.

In Equation (10) are present three "electromagnetic geometric objects": the complex vectors $\widetilde{E}_{p}$ and $\widetilde{\boldsymbol{H}}_{\boldsymbol{q}}$, the complex scalar

$$
\widetilde{\mathcal{P}}=\sum_{p} \widetilde{\boldsymbol{E}}_{p} \odot \widetilde{\boldsymbol{H}}_{p}^{*}
$$

and the complex bivector

$$
\widetilde{\mathcal{D}}=\sum_{p<\boldsymbol{q}}\left(\widetilde{\boldsymbol{E}}_{\boldsymbol{p}} \odot \widetilde{\boldsymbol{H}}_{\boldsymbol{q}}^{*}+\widetilde{\boldsymbol{E}}_{\boldsymbol{q}} \odot \widetilde{\boldsymbol{H}}_{\boldsymbol{p}}^{*}\right)
$$

From $\mathcal{C G}_{n}$ structure, Equation (10) explains the total power energy flow because contains the component $\widetilde{\mathcal{P}}$ related with the power contribution due to frequency-like products and the component $\widetilde{\mathcal{D}}$ associated to the mutual influence of the harmonic components of the fields. Observe that in classical approach this last term vanishes due to the orthogonality of harmonics basis. Nevertheless, a null average value of power without any net energy transferred must be also considered to understand the 
real power flux processes and to fully evaluate the energetic efficiency of electric systems in presence of harmonics.

The above mentioned electromagnetic geometric objects are shown in Figure 3 and must be considered to explain the main aspects of energy transfer quality.
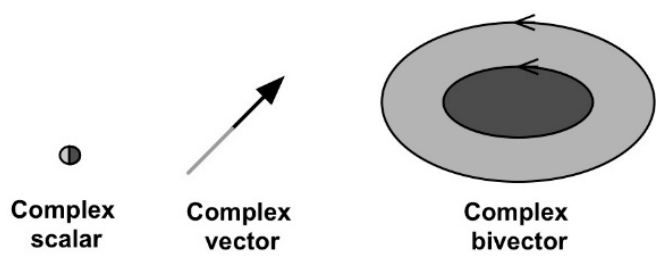

Figure 3. Complex electromagnetic geometric objects.

\section{Formulation of Electromagnetic Energy Quality Index}

\subsection{Energy Flow on Electrical Systems}

Consider the case of an electrical system in the form of a circuit, Figure 4, where the load is a linear/nonlinear system. The supply system voltage is periodic but multi-sinusoidal and can be represented by

$$
u(t)=\sqrt{2} I m \sum_{p} U_{p} e^{j\left(\omega_{p} t+\alpha_{p}\right)}
$$

where $p$ is the harmonic order of $u(t)$. The instantaneous supply current responsible of the generation of the magnetic-field is given by

$$
i(t)=\sqrt{2} \operatorname{Im} \sum_{q} I_{q} e^{j\left(\omega_{q} t+\beta_{q}\right)}
$$

where $q$ is the harmonic order of $i(t)$. It is assumed that a group of voltage harmonics $\mathrm{N}$ exist that have corresponding current harmonics of the same frequencies, and that components $\mathrm{M}$ of current exist without corresponding voltages. In linear operation, $\beta_{q}=\alpha_{q}-\varphi_{q}, \varphi_{q}$ is the impedance phase angle of the consumer electrical system.

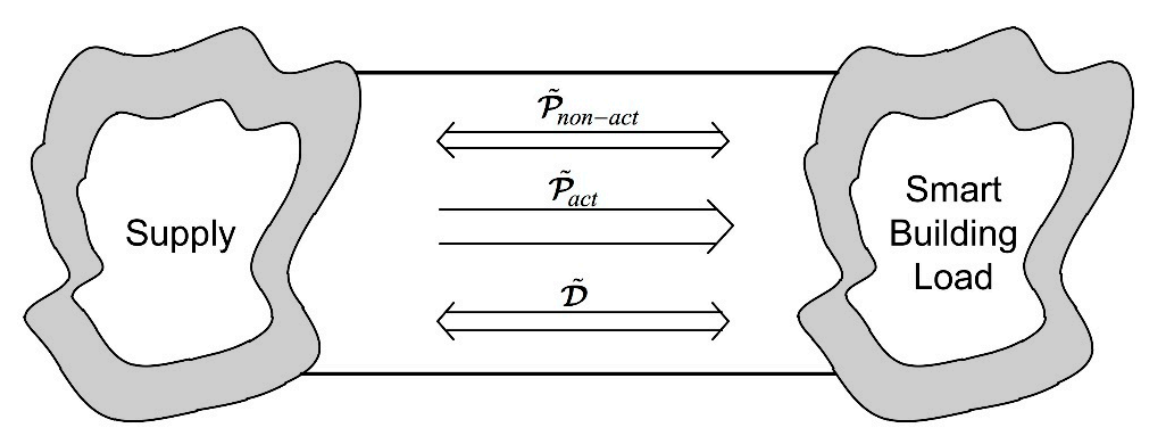

Figure 4. Flux of the generalized Poynting multivector components of electrical systems.

From Equation (10), the energy balance can be expressed as a multivector $\widetilde{S}$ in $\left\{\mathcal{C G}_{n}\right\}$, generated by the geometric product " $\odot$ " of the voltage and conjugate current geometric phasors [14] given by the following set

$$
\widetilde{S}=\widetilde{U} \odot \widetilde{I}^{*}=\widetilde{U} \cdot \widetilde{I}^{*}+\widetilde{U} \wedge \widetilde{I}^{*}
$$

where $\widetilde{U}$ is the voltage geometric phasor and $\widetilde{I}^{*}$ is the conjugated current geometric phasor.

The electromagnetic object $\widetilde{\mathcal{P}}$ can be separated into active and non-active components 


$$
\widetilde{\mathcal{P}}=\sum_{p} \widetilde{\mathcal{P}}_{p_{\text {act }}}+\sum_{p} \widetilde{\mathcal{P}}_{p_{\text {non-act }}}=\underbrace{\operatorname{Re}\left\{\sum_{p} \widetilde{\mathcal{P}}_{p}\right\}}_{\widetilde{\mathcal{P}}_{\text {act }}}+\underbrace{j \operatorname{Im}\left\{\sum_{p} \widetilde{\mathcal{P}}_{p}\right\}}_{\widetilde{\mathcal{P}}_{\text {non-act }}}
$$

where the set $\widetilde{\mathcal{P}}_{\text {act }}$ transfers the harmonic active power and the set $\widetilde{\mathcal{P}}_{\text {non-act }}$ transport the scalar non-active power (classical reactive power). The only component of $\widetilde{\mathcal{P}}$ that transfer useful energy is $\widetilde{\mathcal{P}}_{\text {act }}$, Figure 4 .

Hence, by virtue of (10), it is obtains

$$
\iint_{\zeta} \sum_{p} \overrightarrow{\mathbf{1}}_{Z} \cdot \widetilde{\mathcal{P}}_{p} d \zeta=\iint_{\zeta} \sum_{p} \overrightarrow{\mathbf{1}}_{Z} \cdot \widetilde{\boldsymbol{E}}_{p} \odot \widetilde{\boldsymbol{H}}_{p}^{*} d \zeta=\sum_{P} U_{p} I_{p} e^{j \phi_{p}} \sigma_{0}
$$

The PM $(\widetilde{\mathcal{P}})$ is associated to the energy density at a point on the surface given in terms of the harmonic spatial geometric phasor of electric and magnetic fields at that point. Observe that the PM coincides with the classic Complex Poynting Vector only in pure sinusoidal case.

The real part of $\widetilde{\mathcal{P}}$ in (16), $P=\operatorname{Re}\left\{\iint_{\zeta} \sum_{p} \overrightarrow{\mathbf{1}}_{Z} \cdot \widetilde{\mathcal{P}}_{p} d \zeta\right\}$, permits a direct interpretation in terms of average energy flow, (i.e. active power $\mathrm{P}$ ).

The imaginary part of $\widetilde{\mathcal{P}}$ in (16), $Q=\operatorname{Im}\left\{\iint_{\zeta} \sum_{p} \overrightarrow{\mathbf{1}}_{Z} \cdot \widetilde{\mathcal{P}}_{p} d \zeta\right\}$, is the scalar non-active power. In general, it can be verified that PM $\widetilde{\mathcal{P}}$ considers direction and sense, not only of active power components $\widetilde{\mathcal{P}}_{p_{\text {act }}}$ but also of scalar non-active components $\widetilde{\mathcal{P}}_{p_{\text {non-act }}}$ Thus, the possible reverse sense of any harmonic $\widetilde{\mathcal{P}}_{p_{\text {act }}}$ is very important for a correct identification of harmonic source locations and for determining the responsibility of the utility electrical system and the associated load. Thus, if $\widetilde{\mathcal{P}} p_{\text {act }} \geq 0\left(\boldsymbol{P}_{p} \geq 0\right)$ the energy flow is unidirectional toward the load. On the other way, if $\widetilde{\mathcal{P}}_{p_{\text {act }}} \leq 0\left(\boldsymbol{P}_{\boldsymbol{p}} \leq 0\right)$ the flow is generated in the nonlinearity of the load.

A more detailed develop of the $\mathrm{CPM}(\widetilde{\mathcal{D}})$ is

$$
\begin{aligned}
& \widetilde{\mathcal{D}}=\sum_{p<\boldsymbol{q}} \widetilde{\boldsymbol{E}}_{p} \odot \widetilde{\boldsymbol{H}}_{q}^{*}+\widetilde{\boldsymbol{E}}_{\boldsymbol{q}} \odot \widetilde{\boldsymbol{H}}_{p}^{*}=\sum_{\substack{p<\boldsymbol{q} \\
p, \boldsymbol{q} \in N}} \widetilde{\mathcal{D}}_{p q}+\sum_{p \in N, \boldsymbol{q} \in M} \widetilde{\mathcal{D}}_{p q} \\
& =\underbrace{\operatorname{Re}\left\{\sum_{\substack{p<q \\
p, q \in N}} \widetilde{\mathcal{D}}_{p q}+\sum_{p \in N, q \in M} \widetilde{\mathcal{D}}_{p q}\right\}}_{\widetilde{\mathcal{D}}_{\text {act }}}+j \operatorname{Im}\{\underbrace{\left.\sum_{\substack{p<q \\
p, q \in N}} \widetilde{\mathcal{D}}_{p q}+\sum_{p \in N, q \in M} \widetilde{\mathcal{D}}_{p q}\right\}}_{\widetilde{\mathcal{D}}_{\text {non-act }}}
\end{aligned}
$$

Moreover, the flux of the Complementary Poynting Multivector is given by

$$
\iint_{\zeta} \sum_{p<q} \overrightarrow{\mathbf{1}}_{Z} \cdot \widetilde{\mathcal{D}}_{p q} d \zeta=\widetilde{D}
$$

where $\widetilde{\mathcal{D}}$ can be associated to the polluting nature components, $\widetilde{\mathcal{D}}_{\text {act }}$ and $\widetilde{\mathcal{D}}_{\text {non-act }}$, that do not help in transferring useful energy and

$$
\left.\widetilde{D}=\sum_{\substack{p<\boldsymbol{q} \\ \text { linear }}}\left\{\left(U_{p} I_{q} e^{j \varphi_{q}}-U_{q} I_{p} e^{j \varphi_{p}}\right) e^{j\left(\alpha_{p}-\alpha_{q}\right)}\right)\right\} \sigma_{p q}+\sum_{\substack{p<q \\ \text { non-linear }}} U_{p} I_{q} e^{j\left(\alpha_{p}-\beta_{q}\right)} \sigma_{p q}
$$


is the non-active power bivector called distortion power.

Through (9), (16) and (18), it can be observed that the energy flow that it is originated from the surface of the source in the electrical system equals the flow that enters in the load surface.

\subsection{Electromagnetic Quality Index (EQI)}

The aim of this paper is to propose a multivectorial index $\widetilde{\xi}$, based on electromagnetic geometric objects, capable to characterize the performance of the power transfer or the efficiency of the transmission equipment in single-phase circuits with multi-harmonic signals. This figure of merit can be an important piece of information for the interpretation of the electromagnetic energy flow in presence of disturbing loads. On the contrary, the classic THD index (Total Harmonic Distortion) for the measurement of the harmonic distortion level is not able to give any information about the disturbance nature.

To this end, a new index that integrally reflects the nature of the different energy quality situations is defined. This is expressed as

$$
\widetilde{\zeta}=\frac{\iint_{\zeta} \overrightarrow{\mathbf{1}}_{Z} \widetilde{\Pi} d \zeta}{\operatorname{Re}\left\{\iint_{\zeta} \sum_{p \in N} \overrightarrow{\mathbf{1}}_{Z} \cdot \widetilde{\mathcal{P}}_{p} d \zeta\right\}}=1+j \frac{\operatorname{Im}\left\{\iint_{\zeta} \sum_{p \in N} \overrightarrow{\mathbf{1}}_{Z} \cdot \widetilde{\mathcal{P}}_{p} d \zeta\right\}}{\operatorname{Re}\left\{\iint_{\zeta} \sum_{p \in N} \overrightarrow{\mathbf{1}}_{Z} \cdot \widetilde{\mathcal{P}}_{p} d \zeta\right\}}+\frac{\iint_{\zeta} \sum_{p \neq q} \overrightarrow{\mathbf{1}}_{Z} \cdot \widetilde{\mathcal{D}}_{p q} d \zeta}{\operatorname{Re}\left\{\iint_{\zeta} \sum_{p \in N} \overrightarrow{\mathbf{1}}_{Z} \cdot \widetilde{\mathcal{P}}_{p} d \zeta\right\}}
$$

or also,

$$
\begin{aligned}
& \widetilde{\xi}=\frac{\operatorname{Re}\left\{\iint_{\zeta} \sum_{p=1} \overrightarrow{\mathbf{1}}_{Z} \cdot \widetilde{\mathcal{P}}_{1} d \zeta\right\}+j \operatorname{Im}\left\{\iint_{\zeta} \sum_{p=1} \overrightarrow{\mathbf{1}}_{Z} \cdot \widetilde{\mathcal{P}}_{1} d \zeta\right\}}{\operatorname{Re}\left\{\iint_{\zeta} \sum_{p} \overrightarrow{\mathbf{1}}_{Z} \cdot \widetilde{\mathcal{P}}_{p} d \varsigma\right\}}+ \\
& +\frac{\operatorname{Re}\left\{\iint_{\zeta} \sum_{p \neq 1} \overrightarrow{\mathbf{1}}_{Z} \cdot \widetilde{\mathcal{P}}_{p} d \zeta\right\}+j \operatorname{Im}\left\{\iint_{\zeta} \sum_{p \neq 1} \overrightarrow{\mathbf{1}}_{Z} \cdot \widetilde{\mathcal{P}}_{p} d \zeta\right\}}{\operatorname{Re}\left\{\iint_{\zeta} \sum_{p} \overrightarrow{\mathbf{1}}_{Z} \cdot \widetilde{\mathcal{P}}_{p} d \zeta\right\}}+ \\
& +\frac{\iint_{\zeta} \sum_{p \neq \boldsymbol{q}} \overrightarrow{\mathbf{1}}_{Z} \cdot \widetilde{\mathcal{P}}_{p q} d \zeta}{\operatorname{Re}\left\{\iint_{\zeta} \sum_{p} \overrightarrow{\mathbf{1}}_{Z} \cdot \widetilde{\mathcal{P}}_{p} d \zeta\right\}}
\end{aligned}
$$

The power factor PF can be written as

$$
P F=\frac{1}{\|\widetilde{\tilde{\zeta}}\|}=\frac{\left\|\operatorname{Re}\left\{\iint_{s} \sum_{p} \overrightarrow{\mathbf{1}}_{Z} \cdot \widetilde{\mathcal{P}}_{p} d s\right\}\right\|}{\left\|\iint_{s} \overrightarrow{\mathbf{1}}_{Z} \cdot \widetilde{\Pi} d s\right\|}
$$

It is noteworthy that the power factor is not an exhaustive index for energy quality. In fact, combining (20) and (22) the power factor could be brought to unity, but the electrical systems would still operate in multi-sinusoidal mode.

Equations (21), (22) and (23) show that the index $\widetilde{\xi}$ contains terms that keep direction and sense, allowing harmonic source detection. This property is very important to achieve an appropriated compromise between the energy quality index and the power factor. Moreover, the dominant harmonic source should be based on an evaluation of non-active scalar and bivector electromagnetic geometric object components of the Generalized Poynting Multivector. This subject is discussed in the next subsection. 


\subsection{Illustrative Comparison between Different Non-Active Electromagnetic Geometric Objects}

It is seen from (12) and (17) that $\widetilde{U}_{1}$ and $\widetilde{I}_{1}$ are the sinusoidal voltage and current geometric phasor and $\widetilde{U}_{p}$ and $\widetilde{I}_{q}$ are the harmonic voltage and current geometric phasor when $p \neq 1, q \neq 1$ respectively. Obviously, the voltage geometric phasor $\widetilde{U}_{1}$ produces the sinusoidal electric field spatial geometric phasor $\widetilde{E}_{1}$ and the harmonic voltage $\widetilde{U}_{p}$ generates the harmonic field $\widetilde{E}_{p}$. Similarly the sinusoidal current $\widetilde{I}_{1}$ geometric phasor produces the sinusoidal magnetic field spatial geometric phasor $\widetilde{\boldsymbol{H}}_{1}$ and the harmonic current $\widetilde{I}_{q}$ generates the harmonic field $\widetilde{\boldsymbol{H}}_{q}$. The interaction among these fields produces the cited characteristic electromagnetic geometric objects $\widetilde{\boldsymbol{\Pi}}, \widetilde{\mathcal{P}}$ and $\widetilde{\mathcal{D}}$. The objects $\widetilde{\mathcal{P}}$ and $\widetilde{\mathcal{D}}$ are separated into an active and a non-active component as in Equations (15) and (17). In these equations, the different non-active scalar and bivector electromagnetic objects assume different expressions depending on the electrical system nature.

Starting from these considerations, a new philosophy is proposed for the detection of the dominant harmonic source that is based on the comparison of these non-active components to explain the power quality concept. For more detail, the following situations are considered:

- $\quad$ Sinusoidal case: $p=q=1 \Rightarrow N=\{1\}$

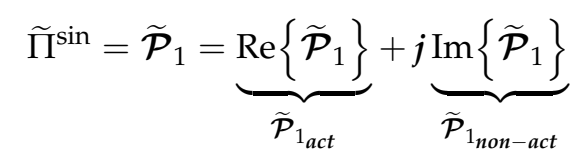

and

$$
\widetilde{\xi}^{\sin }=1+j \frac{\operatorname{Im}\left\{\iint_{\zeta} \sum_{p \in 1} \overrightarrow{\mathbf{1}}_{Z} \cdot \widetilde{\mathcal{P}}_{1} d \varsigma\right\}}{\operatorname{Re}\left\{\iint_{\zeta} \sum_{p \in 1} \overrightarrow{\mathbf{1}}_{Z} \cdot \widetilde{\mathcal{P}}_{1} d \varsigma\right\}}
$$

In this case, the quantity $\operatorname{Im}\left\{\widetilde{\mathcal{P}}_{1}\right\}=\widetilde{\mathcal{P}}_{1_{\text {non-act }}}$ can be considered as minimum reference value to improve the energy quality, since it is the only non-active electromagnetic geometric object. It is the well established fundamental reactive power $(\mathrm{Q})$ that can be reduced to zero by shunts capacitors

$$
\left\|\operatorname{Im}\left\{\iint_{\zeta} \sum_{p \in 1} \overrightarrow{\mathbf{1}}_{\mathbf{Z}} \cdot \widetilde{\mathcal{P}}_{1} d \zeta\right\}\right\|=0
$$

and consequently,

$$
\left\|\widetilde{\xi}^{\sin }\right\|=1 \Rightarrow P F_{1}=1
$$

where $P F_{1}$ is the fundamental power factor, also known as the displacement power factor

$$
P F_{1}=\cos \left(\varphi_{1}\right)
$$

The quantity $\operatorname{Re}\left\{\widetilde{\mathcal{P}}_{1}\right\}=\widetilde{\mathcal{P}}_{1_{\text {act }}}$ transfers the fundamental active power (useful energy) and is associated to the instantaneous active power.

- Multi-sinusoidal linear case: $p \in N, q \in N$ 
If the electrical system is linear, the current requested by the loads and voltage supplied by the mains share the same harmonic order and the equation (8) yields the linear GPM $\widetilde{\Gamma}^{l i n}$

$$
\begin{aligned}
& \widetilde{\Pi}^{\text {lin }}=\sum_{p=q} \widetilde{\mathcal{P}}_{p}+\sum_{p \neq q} \widetilde{\mathcal{D}}_{p q}= \\
& =\underbrace{\sum_{p=q} \widetilde{\mathcal{P}}_{p_{\text {act }}}}_{\widetilde{\mathcal{P}}_{\text {act }}}+\underbrace{\widetilde{\mathcal{P}}_{1_{\text {non-act }}}+\sum_{p, q \in N>1} \widetilde{\mathcal{P}}_{p_{\text {non-act }}}}_{j \widetilde{\mathcal{P}}_{\text {non-act }}}+\underbrace{\sum_{p, q \in N} \widetilde{\mathcal{D}}_{p q}}_{\widetilde{\mathcal{D}}^{\text {lin }}}
\end{aligned}
$$

The linear EQM is given by

$$
\widetilde{\zeta}^{l i n}=1+j \frac{\operatorname{Im}\left\{\iint_{\zeta} \sum_{p \in N} \overrightarrow{\mathbf{1}}_{Z} \cdot \widetilde{\mathcal{P}}_{p} d \zeta\right\}}{\operatorname{Re}\left\{\iint_{\zeta} \sum_{p \in N} \overrightarrow{\mathbf{1}}_{Z} \cdot \widetilde{\mathcal{P}}_{p} d \zeta\right\}}+\frac{\iint_{\zeta} \sum_{p, q \in N} \overrightarrow{\mathbf{1}}_{Z} \cdot \widetilde{\mathcal{D}}_{p q} d \zeta}{\operatorname{Re}\left\{\iint_{\zeta} \sum_{p \in N} \overrightarrow{\mathbf{1}}_{Z} \cdot \widetilde{\mathcal{P}}_{p} d \zeta\right\}}
$$

The quantities $\operatorname{Im}\left\{\iint_{\zeta} \sum_{p \in N} \overrightarrow{\mathbf{1}}_{Z} \cdot \widetilde{\mathcal{P}}_{p} d \zeta\right\}$ and $\operatorname{Im}\left\{\iint_{\zeta} \sum_{p, q \in N} \overrightarrow{\mathbf{1}}_{Z} \cdot \widetilde{\mathcal{D}}_{p q} d \zeta\right\}$ in (29) should be minimized by shunt capacitors or reduced to zero by shunt reactance one-ports. In these conditions

$$
\widetilde{\xi}^{\text {lin }}=1+\frac{\operatorname{Re} \iint_{\zeta} \sum_{p, q \in N} \overrightarrow{\mathbf{1}}_{Z} \cdot \widetilde{\mathcal{D}}_{p q} d \zeta}{\operatorname{Re}\left\{\iint_{\zeta} \sum_{p \in N} \overrightarrow{\mathbf{1}}_{Z} \cdot \widetilde{\mathcal{P}}_{p} d \zeta\right\}} \Rightarrow\left\|\mathfrak{\xi}^{\text {lin }}\right\|>1 \Rightarrow P F<1
$$

From (30), it results that for linear electrical systems under multi-sinusoidal conditions, the criterion

$$
\begin{gathered}
\operatorname{Im}\left\{\iint_{\zeta} \sum_{p \in N} \overrightarrow{\mathbf{1}}_{Z} \cdot \widetilde{\mathcal{P}}_{p} d \zeta\right\}=0 \\
\operatorname{Im}\left\{\iint_{\zeta} \sum_{p, q \in N} \overrightarrow{\mathbf{1}}_{Z} \cdot \widetilde{\mathcal{D}}_{p q} d \zeta\right\}=0
\end{gathered}
$$

does not represent the conditions of highest power factor.

- Multi-sinusoidal non-linear case: $p \in N, q \in N \cup M$

In this case, the presence of the nonlinear loads causes some current components $(q \in M)$ which harmonic orders are not present in the voltage supplied to the electrical system. It is well known that when a non-linear o time-varying electrical system is present, it injects disturbances even if the supply voltage is sinusoidal. In view of (8), the non-linear GPM can be written as follows,

$$
\begin{aligned}
& \widetilde{\boldsymbol{\Pi}}^{\text {non-lin }}=\sum_{p=q} \widetilde{\mathcal{P}}_{p}+\sum_{p \neq q} \widetilde{\mathcal{D}}_{p q}= \\
& =\underbrace{\sum_{p=q} \widetilde{\mathcal{P}}_{p_{\text {act }}}}_{\widetilde{\mathcal{P}}_{\text {act }}}+\underbrace{j \widetilde{\mathcal{P}}_{1_{\text {non-act }}}+\sum_{p, q \in N>1} \widetilde{\mathcal{P}}_{p_{\text {non-act }}}}_{j \widetilde{\mathcal{P}}_{\text {non-act }}}+\underbrace{\sum_{p, q \in N} \widetilde{\mathcal{D}}_{p q}}_{\widetilde{\mathcal{D}}^{\text {lin }}}+\underbrace{\sum_{\substack{p \in N \\
q \in M}} \widetilde{\mathcal{D}}_{p q}}_{\widetilde{\mathcal{D}}^{\text {non-lin }}}
\end{aligned}
$$


The EQI is given by

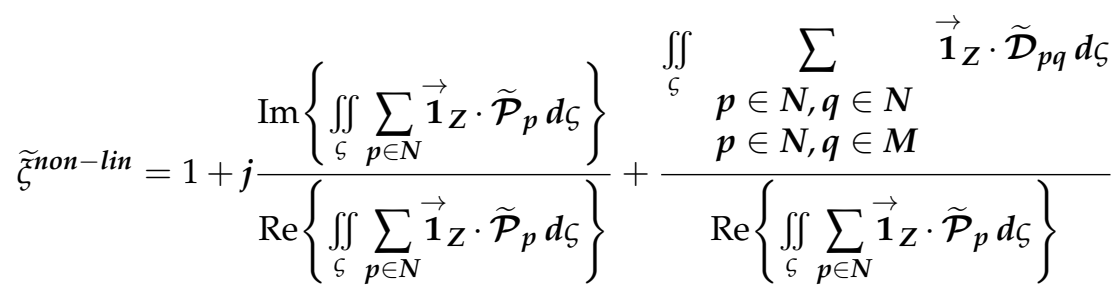

If the equalities (31) and (32) are fulfilled, the Eq. (34) is now given by,

$$
\widetilde{\zeta}^{\text {non-lin }}=1+\frac{\operatorname{Re} \iint_{\zeta} \sum_{p, q \in N} \overrightarrow{\mathbf{1}}_{Z} \cdot \widetilde{\mathcal{D}}_{p q} d \zeta+\iint_{\zeta} \sum_{p \in N, q \in M} \overrightarrow{\mathbf{1}}_{Z} \cdot \widetilde{\mathcal{D}}_{p q} d \zeta}{\operatorname{Re}\left\{\iint_{\zeta} \sum_{p \in N} \overrightarrow{\mathbf{1}}_{Z} \cdot \widetilde{\mathcal{P}}_{p} d \zeta\right\}}
$$

And

$$
\left\|\widetilde{\xi}^{\text {non-lin }}\right\|>1 \Rightarrow P F<1
$$

Comparing the multivectors expressed in (26), (30) and (36), it can be observed that $\widetilde{\zeta}^{\text {non-lin }}$ multivector contains all possible non-active components after passive compensation. Consequently, in the same operation conditions, it can be written that

$$
\left\|\widetilde{\zeta}^{\sin }\right\| \leq\left\|\widetilde{\zeta}^{\text {lin }}\right\| \leq\left\|\widetilde{\xi}^{\text {non-lin }}\right\|
$$

In sinusoidal operation (ideal situation), the three indexes are equal. In the presence of harmonic distortion, the differences among the values of the indexes depend of the supply and of the load characteristics. Then, the possible $\widetilde{\zeta}$ multivectors and their magnitudes depend on the electrical system conditions. Each situation is strictly related to the distortion state of power system and therefore, to the harmonic presence and energy quality, thus, the higher harmonic interaction, the greater non-active energy flow. Observe that the multivectors $\widetilde{\mathcal{P}}_{\text {non-act }}$ and $\widetilde{\mathcal{D}}$ do not help in transferring useful energy and only are associated to the oscillations that produce both, energy lost and stored energy by the loads. Both quantities are related with the non-active power.

As a result of these considerations, an evaluation and comparison of both indexes, $\widetilde{\xi}$ and PF, in the same working conditions, shows that $\widetilde{\xi}$ give two pieces of extra information about the energy quality and the disturbing loads nature.

In conclusion, the suggested EQI possesses clear advantages from the viewpoint of non-active energy flow minimization. The main advantage is that it is decomposed into a complex-scalar and complex-bivectors with direction and sense. These components provide detailed information for a possible minimization of each electromagnetic object term by means of new devices, strategies, and algorithms. The accomplishment of such compensating methods and devices is a problem that warrants further research.

Although the proposed theory is limited to linear and non-linear distorted single-phase power systems, it is worth mentioning that this work does not have in any way the pretension to put an end to the topic, quite the contrary, in fact. Thus, the application of this methodology to polyphase systems deserves in-depth investigations in the near future. This proposed approximation has to face different unsolved problematic aspects. In particular, while the definition of apparent power and related components in balanced three-phase systems with sinusoidal waveforms is well established, the definitions of unbalance conditions are still in an open debate. Moreover, the study of the most general case, the three-phase systems with non-sinusoidal and unbalanced conditions, even needs to improve precedent theories that in many cases are mutually contradictory. 
It is remarkable that the structure of geometric algebra gives a new insight to phase domain and constitutes a powerful tool for the treatment of periodic distorted signals. In this framework, power definition deals with the key concept of geometric phasors, i.e. algebraic time-averaged quantities that are far from being instantaneous ones. In this regard, depending on the considered application, this can be a major restriction. Thus, applications such as instantaneous active filters or fast response compensation devices, are out of the scope of this study, as well. For the same reason, electric signals disturbed with non-stationary events such as transients, sag, swells, etc. are not considered in this work.

\section{Numerical Example}

In order to validate the use and relevance of the Poynting Multivector suggested in this paper, sustainable and smart building loads supplied from a transmission line are analysed in the $\mathcal{C G}_{n}-\mathbb{R}^{3}$ framework. In a simplistic manner, the conductors are considered as rectangular and parallel superconductors meant to facilitate the propagation of energy from source to load, Figure 5 . Units of physical quantities are the standard units of the MKSA system and thus are omitted.

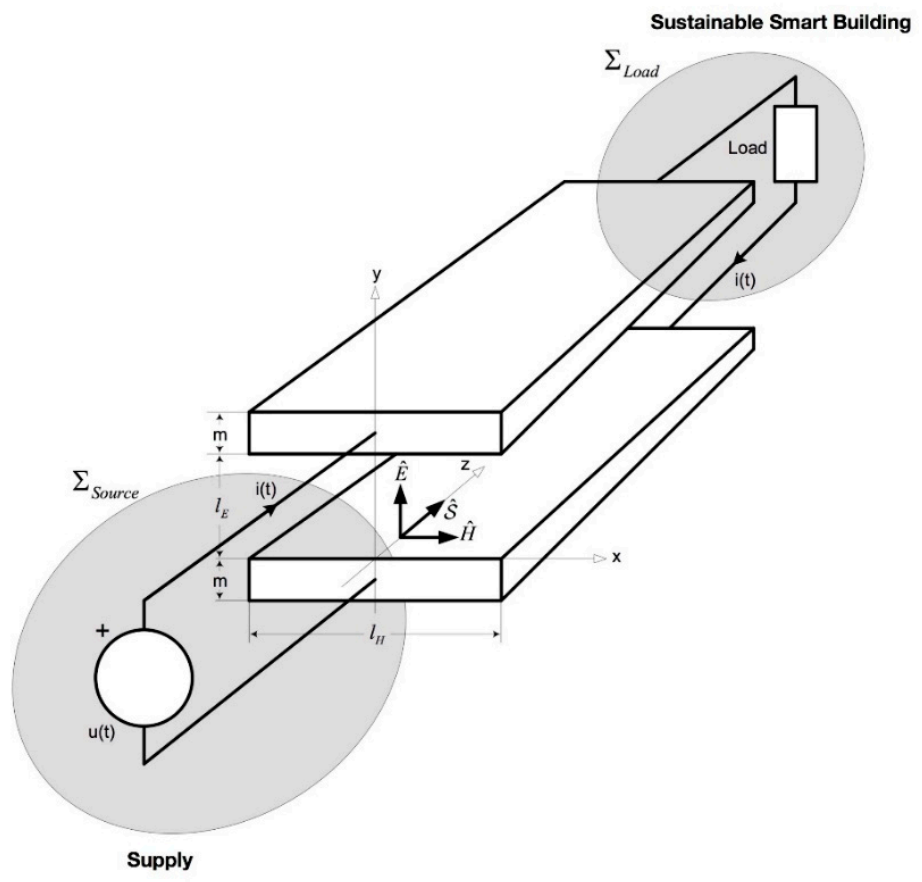

Figure 5. Industrial system: Sustainable smart building.

Two parallel plane conductors in linear media are considered. Both conductors, of thickness $\lambda$ and width $\gamma$, are separated by a dielectric material of thickness $\rho$. We suppose that $\gamma \gg \lambda, \rho$. By ignoring eddy currents, line impedance, fringing effects, three cases are analysed:

\subsection{Linear Load Supplied by A Sinusoidal Voltage Source}

Voltage and a hypothetic resulting current are respectively

$$
\begin{gathered}
u(t)=\sqrt{2}\left(200 \sin \omega_{1} t\right) \\
i(t)=\sqrt{2}\left[10 \sin \left(\omega_{1} t-36.87^{\circ}\right)\right]
\end{gathered}
$$

Then, from [13], the spatial geometric phasors of the electric $\widetilde{\boldsymbol{E}}$ and magnetic $\widetilde{\boldsymbol{H}}^{*}$ fields can be expressed as

$$
\widetilde{\boldsymbol{E}}=\frac{1}{d}\left(200 e^{j 0} \sigma_{1}\right) \overrightarrow{1}_{X} ; \widetilde{\boldsymbol{H}}^{*}=\frac{1}{h}\left(10 e^{j 36.87} \sigma_{1}\right) \overrightarrow{1}_{Y}
$$


and the electromagnetic geometric objects and associated power are

$$
\begin{gathered}
\iint_{\zeta} \sum_{p=1} \overrightarrow{\mathbf{1}}_{Z} \cdot \widetilde{\mathcal{P}} d \zeta=(\underbrace{1600}_{P_{1}}+j \underbrace{1200}_{Q_{1}}) \sigma_{0} \\
\iint_{\zeta} \sum_{p \neq \boldsymbol{q}} \overrightarrow{\mathbf{1}}_{Z} \cdot \widetilde{\mathcal{D}} d \varsigma=0 ; \widetilde{D}=0 \\
\widetilde{\zeta}^{\sin }=1+j \frac{1200}{1600} \sigma_{0} \Rightarrow\left\|\widetilde{\zeta}^{\sin }\right\|=1.25 ; P F=0.8
\end{gathered}
$$

6.2. Linear Load Supplied by A Non-Sinusoidal Sinusoidal Voltage Source

Voltage and hypothetic resulting current are

$$
\begin{gathered}
u(t)=\sqrt{2}\left(200 \sin \omega_{1} t+50 \sin \omega_{2} t\right) \\
i(t)=\sqrt{2}\left[10 \sin \left(\omega_{1} t-36.87^{\circ}\right)+5 \sin \left(\omega_{2} t+53.13^{\circ}\right)\right]
\end{gathered}
$$

and the spatial geometric phasors of the electric $\widetilde{\boldsymbol{E}}$ and magnetic $\widetilde{\boldsymbol{H}}^{*}$ fields can be expressed as

$$
\begin{gathered}
\widetilde{\boldsymbol{E}}=\frac{1}{d}\left(200 e^{j 0} \sigma_{1}+50 e^{j 0} \sigma_{2}\right) \overrightarrow{1}_{X} \\
\widetilde{\boldsymbol{H}}^{*}=\frac{1}{h}\left(10 e^{j 36.87} \sigma_{1}+5 e^{-j 53.13} \sigma_{2}\right) \overrightarrow{1}_{Y} \\
\iint_{\zeta} \sum_{\boldsymbol{p}} \overrightarrow{1}_{Z} \cdot \widetilde{\mathcal{P}} d \zeta=(\underbrace{1750}_{P}+\underbrace{1000}_{Q}) \sigma_{0} \\
\iint_{\zeta} \sum_{\boldsymbol{p} \neq \boldsymbol{q}} \overrightarrow{1}_{Z} \cdot \widetilde{\mathcal{D}} d \zeta=\widetilde{D}=\underbrace{[200-j 1100]}_{\widetilde{D}_{12}} \sigma_{12} \\
\widetilde{\boldsymbol{\zeta}}^{\text {non-lin }}=1+j \frac{1000}{1750} \sigma_{0}+\underbrace{[200-j 1100] \sigma_{12}}_{1750 \sigma_{0}} \\
\left\|\widetilde{\widetilde{\zeta}}{ }^{\text {non }- \text { lin }}\right\|=1.32 \\
P F=0.76
\end{gathered}
$$

6.3. Non-Linear Load Supplied by A Non-Sinusoidal Sinusoidal Voltage Source

Voltage and hypothetic resulting current are

$$
\begin{gathered}
u(t)=\sqrt{2}\left(200 \sin \omega_{1} t+50 \sin \omega_{2} t\right) \\
i(t)=\sqrt{2}\left[10 \sin \left(\omega_{1} t-36.87^{\circ}\right)+5 \sin \left(\omega_{2} t+53.13^{\circ}\right)+5 \sin \left(\omega_{3} t+45^{\circ}\right)\right]
\end{gathered}
$$

and the spatial geometric phasors of the electric $\widetilde{\boldsymbol{E}}$ and magnetic $\widetilde{\boldsymbol{H}}^{*}$ fields can be expressed as

$$
\begin{aligned}
& \widetilde{\boldsymbol{E}}=\frac{1}{d}\left(200 e^{j 0} \sigma_{1}+50 e^{j 0} \sigma_{2}\right) \overrightarrow{1}_{X} \\
& \widetilde{\boldsymbol{E}}=\frac{1}{d}\left(200 e^{j 0} \sigma_{1}+50 e^{j 0} \sigma_{2}\right) \overrightarrow{1}_{X}
\end{aligned}
$$




$$
\begin{gathered}
\iint_{\zeta} \sum_{p} \overrightarrow{1}_{Z} \cdot \widetilde{\mathcal{P}} d \zeta=[(1600+1200)+j(150-200)] \sigma_{0}=(\underbrace{1750}_{P}+j \underbrace{1000}_{Q}) \sigma_{0} \\
\iint_{\zeta} \sum_{p \neq q} \overrightarrow{1}_{Z} \cdot \widetilde{\mathcal{D}} d \zeta=\widetilde{D}=\underbrace{[200-j 1100]}_{\widetilde{D}_{12}} \sigma_{12}+\underbrace{[125 \sqrt{2}-j 125 \sqrt{2}]}_{\widetilde{D}_{23}} \sigma_{13}+\underbrace{[500 \sqrt{2}-j 500 \sqrt{2}]}_{\widetilde{D}_{13}} \sigma_{23} \\
\widetilde{\zeta}^{\text {non-lin }}=1+j \frac{1000}{1750} \sigma_{0}+\frac{[200-j 1100] \sigma_{12}+[125 \sqrt{2}-j 125 \sqrt{2}]}{\sigma_{13}+[500 \sqrt{2}-j 500 \sqrt{2}] \sigma_{23}} \\
\left\|\widetilde{\zeta}^{\text {non }- \text { lin }}\right\|=1.44 \\
P F=0.69
\end{gathered}
$$

As in (37), it should be noticed in this example that

$$
\left\{\left\|\widetilde{\xi}^{\sin }\right\|=1.25\right\}<\left\{\left\|\widetilde{\xi}^{\text {lin }}\right\|=1.32\right\}<\left\{\left\|\widetilde{\xi}^{\text {non-lin }}\right\|=1.44\right\}
$$

Comparing the multivectors $\widetilde{\xi}$ of the above three cases, it can be observed that they contain all possible non-active components. In this way, the possible reverse sense of any harmonic of active, reactive and distortion powers is very important for a correct identification of harmonic source locations, and for determining the responsibility of the utility side (generator) and the consumer side (load) $[20,21]$. Thus, the suggested index $\widetilde{\xi}$ possesses clear advantages from the viewpoint of non-active power flow minimization.

\section{Conclusions}

The analysis of sustainable smart buildings is key to new future buildings, new complex architectures, and its usefulness extends to smart cities. Analyses of quality of the construction typically focus on applying methodologies that evaluate quality objectives at environmental, construction and building levels. Research has shown that a multivector quality index can be useful for detection of harmonic sources of new sustainable smart buildings.

Along this line this paper presents a reformulation of Poynting Vector in terms of the Geometric Algebra framework when inefficiencies caused by harmonics are considered. In this environment, each geometric object represents a different kind of energy flux. Thus, the distinct and well-known power terms in electric power systems, i.e. Active, Reactive, Distortion and Apparent powers acquire a new interpretation and dimension by means of multivectors. This novel approach is applicable to smart architectural single-phase power systems with linear/non-linear loads under sinusoidal or non-sinusoidal conditions. By this means, the behaviour of the energy flux is summarized by the proposed electromagnetic energy index EQI. In addition, the meaning of this original index is deeply analysed and discussed, concluding that its capability to deal with geometric properties, namely magnitude, direction and sense, makes it perfectly appropriate for detection and minimization of harmonic sources.

Furthermore, it is an excellent tool to interpret the cited power flow distribution. Thus, the introduction of this figure of merit supposes not only a generalization of the traditional power factor in electric power networks but also the key to the electromagnetic energy transfer between mains and loads. The global efficiency of a system is truth evaluated by this index because it includes all possible interactions.

It is remarkable that traditional Poynting Vector is a particular case of the proposed generalized Poynting Multivector when no cross interactions are present between different harmonics. This correlation is now precisely understood as the outer product between geometric phasors of different energy levels. Thus, this fact is crucial for future research proposals on the design of passive 
and/or active architectures for selective compensation, optimization algorithms and measurement techniques for harmonic pollution monitoring.

The electromagnetic nature of the power components could be the key that opens a broad range of new interesting research lines in electrical engineering and all energy efficiency related matters.

Author Contributions: All authors contributed equally to this work. All authors wrote, reviewed and commented on the manuscript. All authors have read and approved the final manuscript.

Conflicts of Interest: The authors declare no conflict of interest.

\section{Glossary of Symbols}

$\mathbb{R} \quad$ real numbers

$\mathcal{C} \quad$ complex vector space

$\mathcal{G}_{n} \quad$ Geometric Algebra in $n$-dimensional real space

$\mathcal{C G}_{n} \quad$ Complex Geometric Algebra

$\mathcal{C G}_{n}^{t}-\mathbb{R}^{3} \quad$ time generalized geometric euclidean space

$\mathcal{C G}_{n}-\mathbb{R}^{3} \quad$ frequency generalized geometric euclidean space

$\overrightarrow{\mathbf{1}}_{X}, \overrightarrow{\mathbf{1}}_{Y}, \overrightarrow{\mathbf{1}}_{Z} \quad$ Euclidean canonical basis

$\sigma_{1 \ldots k} \quad$ canonical basis of $\mathcal{G}_{n}$

$\sigma_{p} \quad$ basis vector of $\mathcal{G}_{n}$

$\sigma_{p} \sigma_{q}=\sigma_{p q} \quad$ basis bivectors of $\mathcal{G}_{n}$

$\sigma_{1} \sigma_{2} \sigma_{3} \quad$ trivector or pseudoscalar of $\mathcal{G}_{n}$

$\lambda, \mu \quad$ scalars or 0-grade geometric object

$a, b \quad$ vectors or 1-grade geometric object

$B \quad$ bivector or 2-grade geometric object

$J$

$\widetilde{M}$

generalized Poynting multivector (GPM)

$\widetilde{\mathcal{P}} \quad$ Poynting multivector (PM)

$\widetilde{\mathcal{D}} \quad$ Complementary Poynting multivector (CPM)

$\widetilde{E} \quad$ electric field geometric phasor

$\widetilde{\boldsymbol{H}} \quad$ magnetic field geometric phasor

$\odot \quad$ generalized geometric product

- inner product

$\wedge \quad$ outer product

j $\quad$ imaginary unit

* $\quad$ conjugated operation

$+\quad$ reverse operation

$\sim \quad$ multivector characterization

$U_{p} \quad p$-th harmonic voltage rms value

$I_{q} \quad q$-th harmonic current rms value

$P \quad$ active power or real part of 0-grade power multivector

$Q \quad$ reactive power or imaginary part of 0 -grade power multivector

$D \quad$ distortion power or 2-grade power

$\widetilde{S} \quad$ power multivector

$\|\widetilde{S}\|, S \quad$ apparent power multivector

$\omega_{\mathrm{p}} \quad p$-th harmonic frequency

$\alpha_{\mathrm{p}} \quad$ phase angle of $p$-th voltage geometric phasor

$\beta_{\mathrm{q}} \quad$ phase angle of $q$-th current geometric phasor

$\phi_{\mathrm{q}} \quad q$-th impedance phase angle

$\widetilde{\xi} \quad$ electromagnetic quality index multivector $(E Q I)$

$\mathrm{PF} \quad$ power factor 


\section{References}

1. Ibrahim, M.; El-Zaart, A.; Adams, C. Smart Sustainable Cities roadmap: Readiness for transformation towards urban sustainability. Sustain. Cities Soc. 2018, 37, 530-540. [CrossRef]

2. Höjer, M.; Wangel, J. Smart sustainable cities: Definition and challenges. In ICT Innovations for Sustainability; Hilty, L., Aebischer, B., Eds.; Springer: Cham, Switzerland, 2015; pp. 333-349.

3. Pervez, H.S.; Nursyarizal, B.M.N.; Perumal, N.; Irraivan, E.; Taib, I. A review on optimized control systems for building energy and comfort management of smart sustainable buildings. Renew. Sustain. Energy Rev. 2014, 34, 409-429. [CrossRef]

4. Nardi, I.; Lucchi, E.; Rubeis, T.; Ambrosini, D. Quantification of heat energy losses through the building envelope: A state-of-the-art analysis with critical and comprehensive review on infrared thermography. Build. Environ. 2018, 146, 190-205. [CrossRef]

5. Karmellos, M.; Kiprakis, A.; Mavrotas, G. A multi-objective approach for optimal prioritization of energy efficiency measures in buildings: Model, software and case studies. Appl. Energy. 2015, 139, 131-150. [CrossRef]

6. Poynting, J.H. On the transfer of energy in the electromagnetic field. Philos. Trans. R. Soc. 1884, 175, 343-361. [CrossRef]

7. Steinmetz, C.P. Theory and Calculation of Alternating Current Phenomena; McGraw Publishing Company: New York, NY, USA, 1900.

8. Emanuel, A.E. Powers in nonsinusoidal situations a review of definitions and physical meaning. IEEE Trans. Power Deliv. 1990, 5, 1377-1389. [CrossRef]

9. Emanuel, A.E. Poynting vector and the physical meaning of nonactive powers. IEEE Trans. Instrum. Meas. 2005, 54, 1457-1462. [CrossRef]

10. Lundin, U.; Bolund, B.; Leijon, M. Poynting vector analysis of synchronous generators using field simulations. IEEE Trans. Magn. 2007, 43, 3601-3606. [CrossRef]

11. Faria, J.A.B. The role of Poynting's vector in polyphase power calculations. Eur. Trans. Electr. Power 2009, 19, 683-688. [CrossRef]

12. De León, F.; Cohen, J. AC power theory from Poynting Theorem: Accurate identification of instantaneous power components in nonlinear-switched circuits. IEEE Trans. Power Deliv. 2010, 25, 2104-2112. [CrossRef]

13. Cheng, P.; Yang, F.; Luo, H.; Guo, H.; Ran, W.; Yang, Y.; Ullah, I. A method to calculate the reactive power of iced transmission line based on Poynting vector and FDFD. Int. J. Appl. Electromagn. Mech. 2016, 50, 417-433. [CrossRef]

14. Emanuel, A.E. About the Rejection of Poynting Vector in Power Systems Analysis. Electr. Power Qual. Util. J. 2007, 13, 43-49.

15. Emanuel, A.E. Power Definitions and the Physical Mechanism of Power Flow; Wiley: Hoboken, NJ, USA, 2010.

16. Todeschini, G.; Emanuel, A.E.; Ferrero, A.; Morando, A.P. A Poynting vector approach to the study of the Steinmetz compensator. IEEE Trans. Power Deliv. 2007, 22, 1830-1833. [CrossRef]

17. Ferrero, A.; Leva, S.; Morando, A.P. An approach to the non-active power concept in terms of the poynting-park vector. Eur. Trans. Electr. Power 2001, 11, 291-299. [CrossRef]

18. Czarnecki, L.S. Considerations on the Concept of Poynting Vector Contribution to Power Theory Development. In Proceedings of the 6th International Workshop on Power Definitions and Measurements Under Nonsinusoidal Conditions, Milano, Italy, 13-15 October 2003.

19. Czarnecki, L.S. Could Power Properties of Three-Phase Systems Be Described in Terms of the Poynting Vector? IEEE Trans. Power Deliv. 2006, 21, 339-344. [CrossRef]

20. Balci, M.; Hocaoglu, M.; Power, S.A. Transition from Poynting vector to instantaneous power. In Proceedings of the 12th International Conference on Harmonics and Quality of Power, Cascais, Portugal, 1-5 October 2006.

21. Stahlhut, J.W.; Browne, T.J.; Heydt, G.T. The Assessment of the Measurement of the Poynting Vector for Power System Instrumentation. In Proceedings of the 39th North American Power Symposium, Las Cruces, NM, USA, 30 September-2 October 2007; pp. 237-241.

22. Castilla, M.; Bravo, J.C.; Ordonez, M.; Montano, J.C. An approach to the multivectorial apparent power in terms of a generalized poynting multivector. Prog. Electromagn. Res. B 2009, 15, 401-422. [CrossRef]

23. Clifford, W.K. Applications of Grassmann's Extensive Algebra. Am. J. Math. 1878, 1, 350. [CrossRef] 
24. Hestenes, D. A unified language for mathematics and physics. their Appl. Math. Phys. 1986. [CrossRef]

25. Chappell, J.M.; Drake, S.P.; Seidel, C.L.; Gunn, L.J.; Iqbal, A.; Allison, A.; Abbott, D. Geometric algebra for electrical and electronic engineers. Proc. IEEE 2014. [CrossRef]

26. Doran, C.; Lasenby, A. Geometric Algebra for Physicists; Cambridge University Press: Cambridge, UK, 2009.

27. Castilla, M.; Bravo, J.C.; Ordóñez, M.; Montaño, J.C. Clifford theory: A geometrical interpretation of multivectorial apparent power. IEEE Trans. Circuits Syst. I Regul. Pap. 2008, 55, 3358-3367. [CrossRef]

28. Castilla, M.; Bravo, J.C.; Ordonez, M.; Montaño, J.C.; Lopez, A.; Borras, D.; Gutierrez, J. The geometric algebra as a power theory analysis tool. In Proceedings of the 2008 International School on Nonsinusoidal Currents and Compensation, Lagow, Poland, 10-13 June 2008.

29. Castilla, M.; Bravo, J.C.; Ordóñez, M. Geometric algebra: A multivectorial proof of Tellegen's theorem in multiterminal networks. IET Circuits, Devices Syst. 2008, 2, 383-390. [CrossRef]

30. Bravo, J.C.; Castilla, M.V. Energy Conservation Law in Industrial Architecture: An Approach through Geometric Algebra. Symmetry 2016, 8, 92. [CrossRef]

31. Castro-Núñez, M.; Londoño-Monsalve, D.; Castro-Puche, R.M. the conservative power quantity based on the flow of energy. J. Eng. 2016, 2016, 269-276. [CrossRef]

32. Petroianu, A.I. A geometric algebra reformulation and interpretation of Steinmetz's symbolic method and his power expression in alternating current electrical circuits. Electr. Eng. 2015, 97, 175-180. [CrossRef]

33. Menti, A.; Zacharias, T.; Milias-Argitis, J. Geometric Algebra: A Powerful Tool for Representing Power Under Nonsinusoidal Conditions. IEEE Trans. Circuits Syst. I Regul. Pap. 2007, 54, 601-609. [CrossRef]

34. Castro-Nunez, M.; Castro-Puche, R. Advantages of Geometric Algebra Over Complex Numbers in the Analysis of Networks With Nonsinusoidal Sources and Linear Loads. IEEE Trans. Circuits Syst. I Regul. Pap. 2012, 59, 2056-2064. [CrossRef]

35. Herrera, R.S.S.; Salmeron, P.; Vazquez, J.R.R.; Litran, S.P.P.; Perez, A. Generalized instantaneous reactive power theory in poly-phase power systems. In Proceedings of the 13th European Conference on Power Electronics and Applications, Barcelona, Spain, 8-10 September 2009; pp. 1-10.

(C) 2018 by the authors. Licensee MDPI, Basel, Switzerland. This article is an open access article distributed under the terms and conditions of the Creative Commons Attribution (CC BY) license (http:/ / creativecommons.org/licenses/by/4.0/). 